\section{READER'S FORUM}

Won-Young Park, Min Soo Kim, Min-Seok Kim, MinHee Oh, Su-Young Lee, Sun-Hun Kim, Jin-Hyoung Cho

Effects of pre-applied orthodontic force on the regeneration of periodontal tissues in tooth replantation.

- Korean J Orthod 2019;49:299-309

The authors' efforts in doing a complicated experiment to find the biologic effect of pre-applied orthodontic force on the regeneration of periodontal ligament (PDL) tissues for tooth replantation procedure were appreciated. For better understanding of mine as a reader and an orthodontic clinician, I would like to ask some questions.

Q1. The authors luxated the teeth and replanted them in their original sockets. In this case, the donor site is the recipient site. That means that the preapplied orthodontic force might affect the donor site, the recipient site, or both at the same time. Could you please explain more in detail why you could interpret the result more focused at the effect on the donor site rather than the recipient site?

Q2. Would you please suggest a clinical protocol for preparing the donor and/or recipient site for auto-transplantation in a stepwise manner, which can be applicable to real treatment? Also, please discuss how much force and in which direction is recommended and how long we should wait after each step until proceeding to the next.
Q3. According to your interests and experience, if there are any, please share with us your opinion on how to make up a team to accomplish the successful auto-transplantation.

Questioned by

ll-Hyung Yang

Department of Orthodontics, School of Dentistry, Seoul National University, Seoul, Korea

A1. What you point out is perfectly correct. 1 think that pre-applied orthodontic force not only affected the PDL of experimental teeth, but also affected the periodontal tissue, including the surrounding alveolar bone.

When planning this experiment for the first time, we tried to do auto-transplantation, not replantation. But, we concluded that it was impossible to do it, given the limitations of the number and form of the 1st molar of rat. Subsequently, we conducted the experiment with replantation, and all the related studies were inferred from the results through replantation.

However, 1 believe that what 1 want to see in this study can be similar, whether replantation or transplantation, because 1 have seen results that were limited to the response and differentiation of the PDL, not the surrounding alveolar bone. Nevertheless, as you pointed out, 1 don't think we can completely rule out the effects of the recipient site in the teeth.

A2. The first thing to do is, of course, to apply the orthodontic force, which does not necessarily require the use of a fixed appliance, such as braces. Applied orthodontic force in two to four weeks will result in sufficient degree of mobility of teeth and activation of PDL cell. 
The second stage is the transplant of the teeth, where three-dimensional printing of the donor tooth can be useful from computed tomography or cone beam computed tomography data. This makes it easy to adjust the form of recipient site, and reduce the out-of-socket time and the damage of donor tooth. The minimal fixation of transplanted teeth is recommended to modern trends.

For the last step, root canal treatment shall be performed. For those who want more information in this aspect of the study, 1 would recommend Cho et al. ${ }^{1}$

A3. In my experience, we need basically orthodontists, conservative dentists or endodontists, and oral surgeons or periodontists. Furthermore, 1 believe that oral and maxillofacial radiologists will also be helpful for evaluation and progress. And of course, a general discussion of the patient and the order in which the treatment progress is made should be understood by all team members.

Replied by

Jin-Hyoung Cho

Department of Orthodontics, School of Dentistry and Dental 4D Research Institute, Chonnam National University, Gwangju, Korea

\section{Reference}

1. Cho JH, Hwang HS, Chang HS, Hwang YC. Application of orthodontic forces prior to autotransplantation case reports. Int Endod J 2013;46:187-94. 